\title{
Epidemiology of Viruses Causing Pediatric Community Acquired Pneumonia in Shanghai During 2010-2020: What Happened Before and After the COVID-19 Outbreak?
}

Fei Li · Yuhan Zhang $\cdot$ Peng Shi $\cdot$ Linfeng Cao $\cdot$ Liyun Su

Yulan Zhang $\cdot$ Ke Peng $\cdot$ Roujian Lu $\cdot$ Wenjie Tan $\cdot$ Jun Shen (D)

Received: September 5, 2021 / Accepted: October 8, 2021 / Published online: October 25, 2021

(c) The Author(s) 2021

\begin{abstract}
Introduction: Since the global outbreak of COVID-19, there has been a significant reduction in pediatric outpatient and emergency visits for infectious diseases. The purpose of this study was to analyze the changes in respiratory viruses in children with community-acquired pneumonia (CAP) in Shanghai in the past 10 years, especially in the first year after COVID19.

Methods: We conducted a retrospective, observational study; the results for eight common respiratory viruses (respiratory syncytial virus (RSV), influenza virus $A$ and $B$, parainfluenza virus 1-3 (PIV), adenovirus (ADV) and human metapneumovirus) tested by direct fluorescent antibody assays in hospitalized CAP
\end{abstract}

Fei Li and Yuhan Zhang contributed equally to this article.

F. Li · Y. Zhang · P. Shi · L. Cao · L. Su · J. Shen ( $ه)$ Children's Hospital of Fudan University, National Children's Medical Center, Shanghai, China e-mail: echoshen11@163.com

Y. Zhang · K. Peng

Wuhan Institute of Virology, Chinese Academy of Sciences, Wuhan, China

R. Lu $\cdot$ W. Tan $(\bowtie)$

National Institute for Viral Disease Control and Prevention, China CDC, Beijing, China e-mail: tanwj28@163.com cases in Children's Hospital of Fudan University during 2010-2020 were analyzed.

Results: Of the 5544 hospitalized CAP patients included in this study, 20.2\% (1125/5544) were positive for the eight respiratory viruses. The top three pathogens were RSV, PIV3 and ADV, detected from $9.8 \%(543 / 5544), 5.3 \%$ (294/ $5544)$ and $2.0 \%(111 / 5544)$ of the samples, respectively. RSV had the highest positive rates among children $<2$ years old. In 2020, the detection rate of all viruses showed a sharp decline from February to August compared with the previous 9 years. When the Shanghai community reopened in August 2020, the detection rate of eight viruses rebounded significantly in September.

Conclusions: These eight respiratory viruses, especially RSV and PIV, were important pathogens of CAP in Shanghai children in the past 10 years. The COVID-19 pandemic had a significant impact on the detection rates for eight respiratory viruses in children with CAP in Shanghai.

Keywords: COVID-19; Children; Pneumonia; Respiratory viruses 


\section{Key Summary Points}

1. The average detection rate of the eight common respiratory viruses by DFA was $20.3 \%$ in children with communityacquired pneumonia (CAP) in Shanghai during the past 10 years.

2. There was a sharp drop in the detection rate for each virus in 2020.

3. After the community reopened, the detection rate of the eight respiratory viruses showed a perfect V-shaped increase.

4. The changes in social hygiene practices triggered by the COVID-19 pandemic had an unimaginable impact on the decline of respiratory virus prevalence among children.

5. This phenomenon has important implications for the control of a new or reemerging respiratory virus in the population in the future.

\section{INTRODUCTION}

Significant changes in pathogen detection in patients with respiratory infections including CAP due to the outbreak of severe acute respiratory syndrome coronavirus 2 (SARS-CoV-2) were reported in late 2019 [1-6]. These changes might have come from the competition of SARS-CoV-2 with other respiratory viruses, but they were more likely to have come from improvements in population hygiene practices to prevent SARS-CoV-2 transmission, such as wearing facemasks, paying attention to hand hygiene and keeping social distance [7]. In some ways, the COVID-19 pandemic is a great opportunity for us to learn how to control the prevalence of both regular and emerging respiratory viruses in the population [8-11].

Children are the main groups in the population to establish herd immunity against circulating respiratory and enteroviruses. They can also reflect, to some extent, the prevalence of various major respiratory and enteroviruses in communities during the COVID-19 era. Sudden changes in people's hygiene practices and strict epidemic prevention and control policies might have a huge impact on the "normal" prevalence of respiratory and gastrointestinal infectious diseases in the population, but we lacked sufficient data to support that assumption. There have been no real-world studies on how the prevalence of infectious diseases rebound in a community as the pressure to change hygiene habits and behaviors is removed.

Here, we describe a retrospective study of 10 years (2010-2020) of DFA test results in children hospitalized with CAP in Children's Hospital of Fudan University and present the dramatic changes in these results triggered by social hygiene practices and government policy changes since January 2020 in Shanghai.

This study involving human participants was reviewed and approved by the medical ethics committee of the Children's Hospital of Fudan University (2021-292).

\section{METHODS}

\section{Patients and Data Collection}

This retrospective study included all hospitalized patients in Children's Hospital of Fudan University diagnosed with CAP and admitted: (1) every Monday between September 1, 2010, and December 31, 2019; (2) every Monday and Wednesday between January 1, 2020, and December 31, 2020.

Patients with nosocomial respiratory infections, neonates, patients with inhaled airway foreign bodies and those with tuberculosis infections were excluded. Criteria for severity of CAP were based the diagnostic criteria recommended by the 2011 Infectious Diseases Society of America community-acquired pneumonia management guideline [12].

To better evaluate the changes in the detection of respiratory and gastrointestinal viruses before and after 2020, we counted all cases 


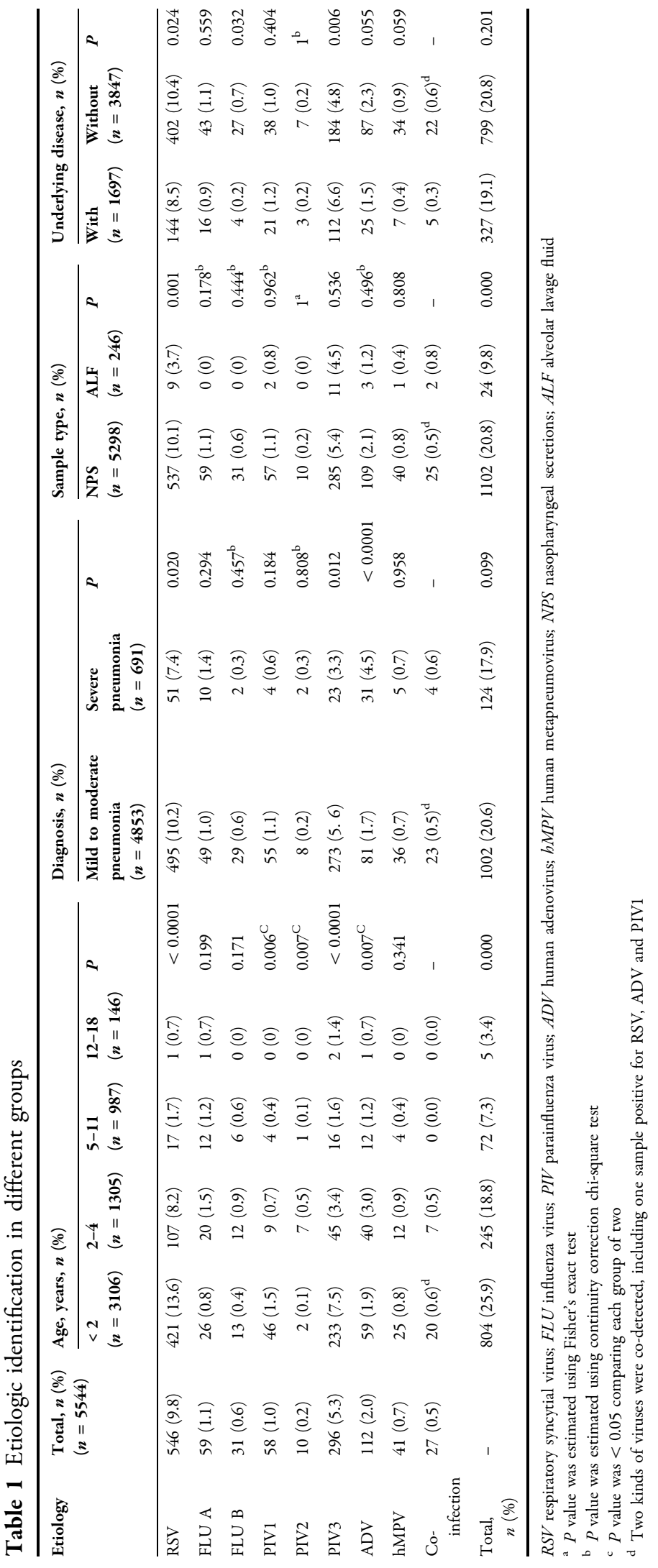




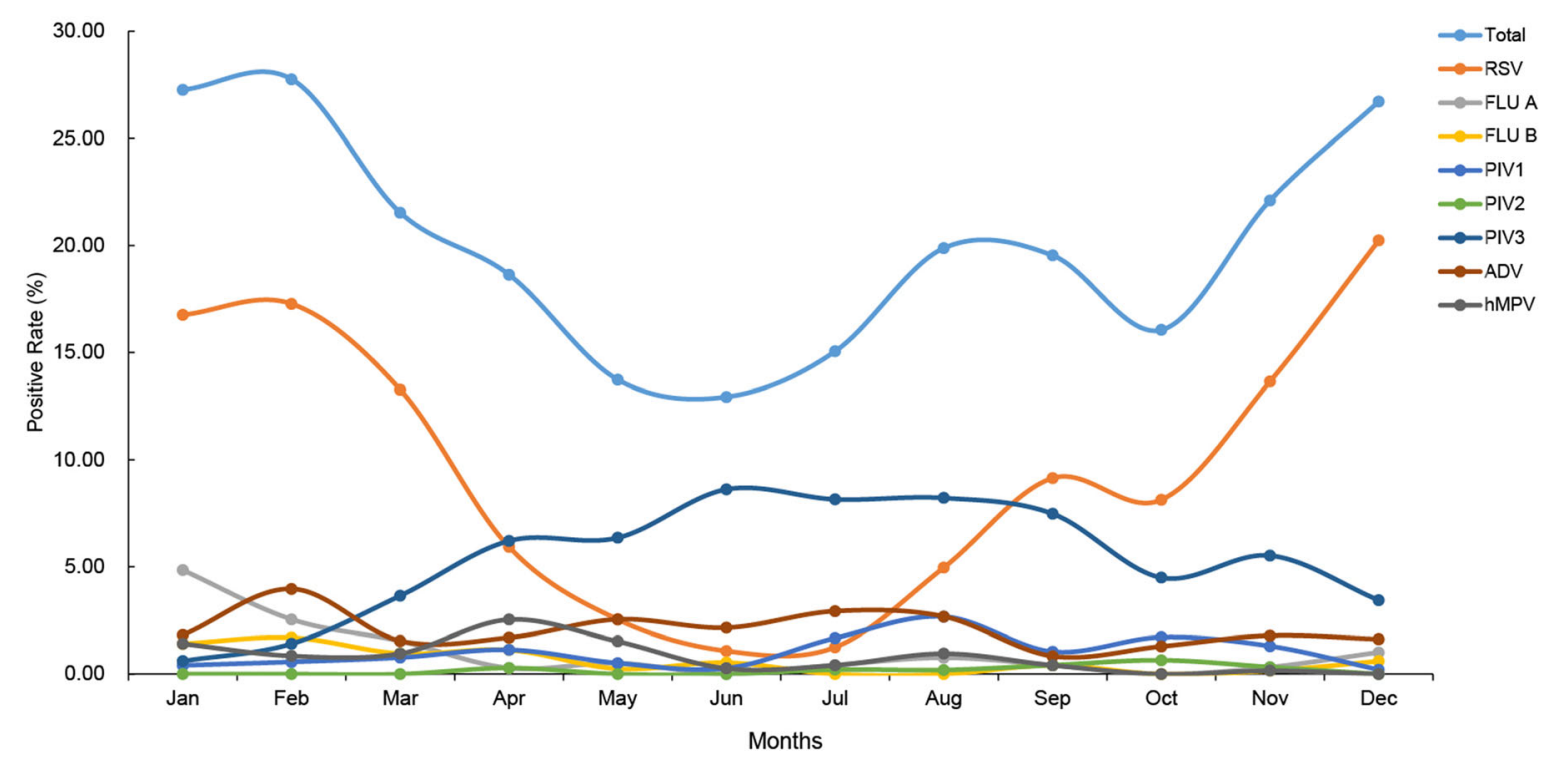

Fig. 1 Distribution of virus detection rate in each month of 2010-2020

diagnosed with hand-foot-mouth disease (HFMD) or herpangina between July 1, 2019, and December 31, 2020, from our hospital's electronic medical history system database.

\section{Virus Detection}

Nasopharyngeal secretions (NPS) or alveolar lavage fluids (ALF) of all CAP patients were routinely collected within $24 \mathrm{~h}$ of admission and detected by direct fluorescent antibody (DFA) assays in our hospital. Respiratory syncytial virus (RSV), influenza virus A and B (FLU A/B), parainfluenza virus 1-3 (PIV1-3) and adenovirus (ADV) were detected by a commercial DFA assay (Chemicon International Inc., Temecula, CA, USA); human metapneumovirus (hMPV) was screened by another DFA assay (Diagnosis Hybrids Inc., USA).

\section{Statistical Analysis}

Continuous variables were presented as mean \pm SD standard deviation (SD) and compared between groups by Student's $t$-test when normally distributed. For not normally distributed variables, the data were expressed as median (interquartile range, IQR), and nonparametric tests were used. Categorical variables were expressed as number (\%) and compared between groups by $\chi^{2}$ or Fisher's exact tests, when appropriate. IBM SPSS Statistics 22.0 was used for data analysis. The significance level was set at $P<0.05$.

\section{RESULTS}

A total of 5627 samples from 5627 cases were studied. Excluded patients included 10 cases of nosocomial respiratory infections, 70 neonates, 2 patients with inhaled airway foreign bodies and 1 patient with suspected tuberculosis. We enrolled 5544 samples collected from 5544 CAP patients in total. The median age of the enrolled patients was 1.0 (IQR 0.5-4.0) years at the time of diagnosis; $56.0 \%(3106 / 5544)$ of the patients were $<2$ years old. Overall, $87.5 \%(4853 / 5544)$ patients had mild to moderate pneumonia and $12.4 \%(691 / 5544)$ severe pneumonia. Of these patients, 30.6\% (1697/5544) had underlying disease, including congenital heart disease, epilepsy, immunodeficiency disease and others.

In total, $20.3 \%(1126 / 5544)$ cases were positive for at least one of the eight respiratory 


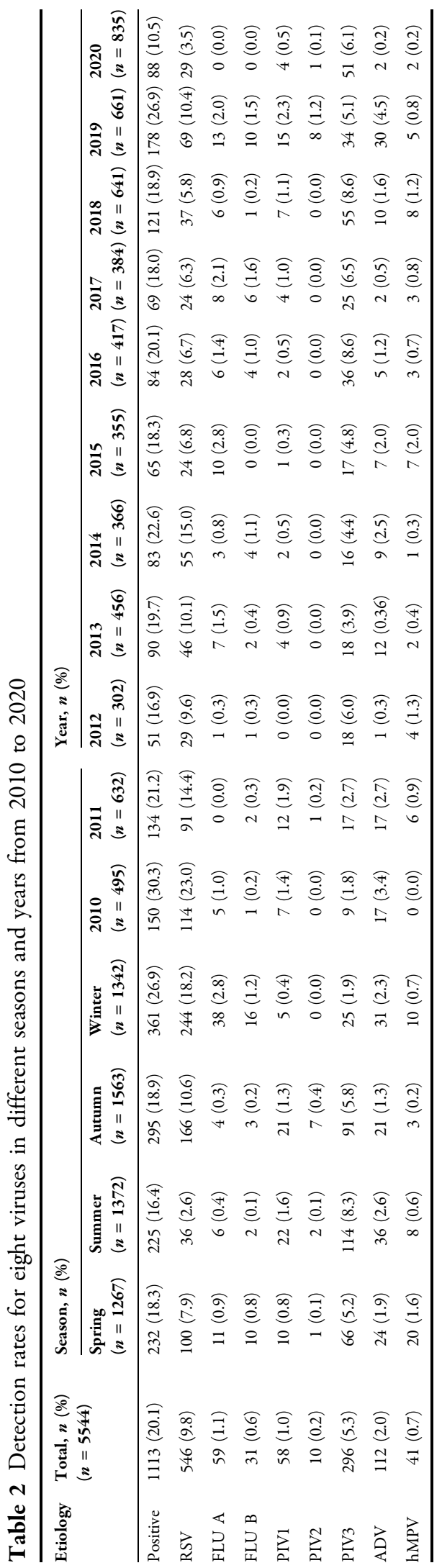

viruses, with $0.4 \%(27 / 5544)$ being co-positive. The top three pathogens were RSV, PIV3 and ADV, detected from $9.8 \%(543 / 5544), 5.3 \%$ (294/5544) and $2.0 \%(111 / 5544)$ of the samples, respectively. RSV and PIV3 had the highest positive rates among children $<2$ years old $(P<0.001)$; ADV had the highest positive rate $(3.0 \%, 40 / 1305)$ in children aged 2-4 years (Table 1). Regarding disease severity, $12.4 \%$ (691/5544) of the patients had severe pneumonia. Of the severe cases, $17.9 \%$ (124/691) were positive for the eight viruses, lower than the positive rate of $20.6 \%(1002 / 4853)$ in mild to moderate pneumonia cases $(P=0.099)$. We found $4.5 \%$ (31/691) of the severe pneumonia cases were positive for ADV, higher than for cases with mild to moderate pneumonia $(P<0.001)$, while the other seven viruses were not. As for different sample types, RSV had a higher positive rate in NPS $(10.1 \%, 537 / 5298)$ than in ALF $(3.7 \%, 9 / 246) \quad(P=0.001)$, and patients with underlying disease had lower positive rates of $\operatorname{RSV}(8.4 \%, 144 / 1697)$ than those without $(10.4 \%, 402 / 3847) \quad(P=0.024)$ (Table 1).

All eight respiratory viruses could be detected each month. The distribution of the total detection rate for the eight viruses by month showed a "canyon" pattern during the past 10 years, with the bottom in July to August when the temperature was highest in Shanghai (Fig. 1). Of the samples, $27.7 \%(98 / 353)$ were positive in February and only $12.9 \%$ (48/371) in June. The RSV detection rate showed a nearly perfect U-shaped pattern for the past 10 years. Contrarily, the detection rates of PIV3 and ADV were significantly increased in the months with higher temperature in Shanghai (Fig. 1, Table 2).

Figure $2 \mathrm{~A}$ shows the total detection rate for the eight viruses (range: 1.3-38.8\%), presenting obvious seasonality. Significant differences existed in the overall positive rates of these viruses in winter $(27.1 \%, 365 / 1342)$ compared with spring $(18.3 \%, 232 / 1267)$, summer $(16.3 \%$, 224/1372) and autumn (19.5\%, 305/1563) $(P=0.000)$ (Table 2$)$, which was mainly due to the contribution of the high RSV detection rate in winter. There was a peak of RSV detection every winter (December to February of the 

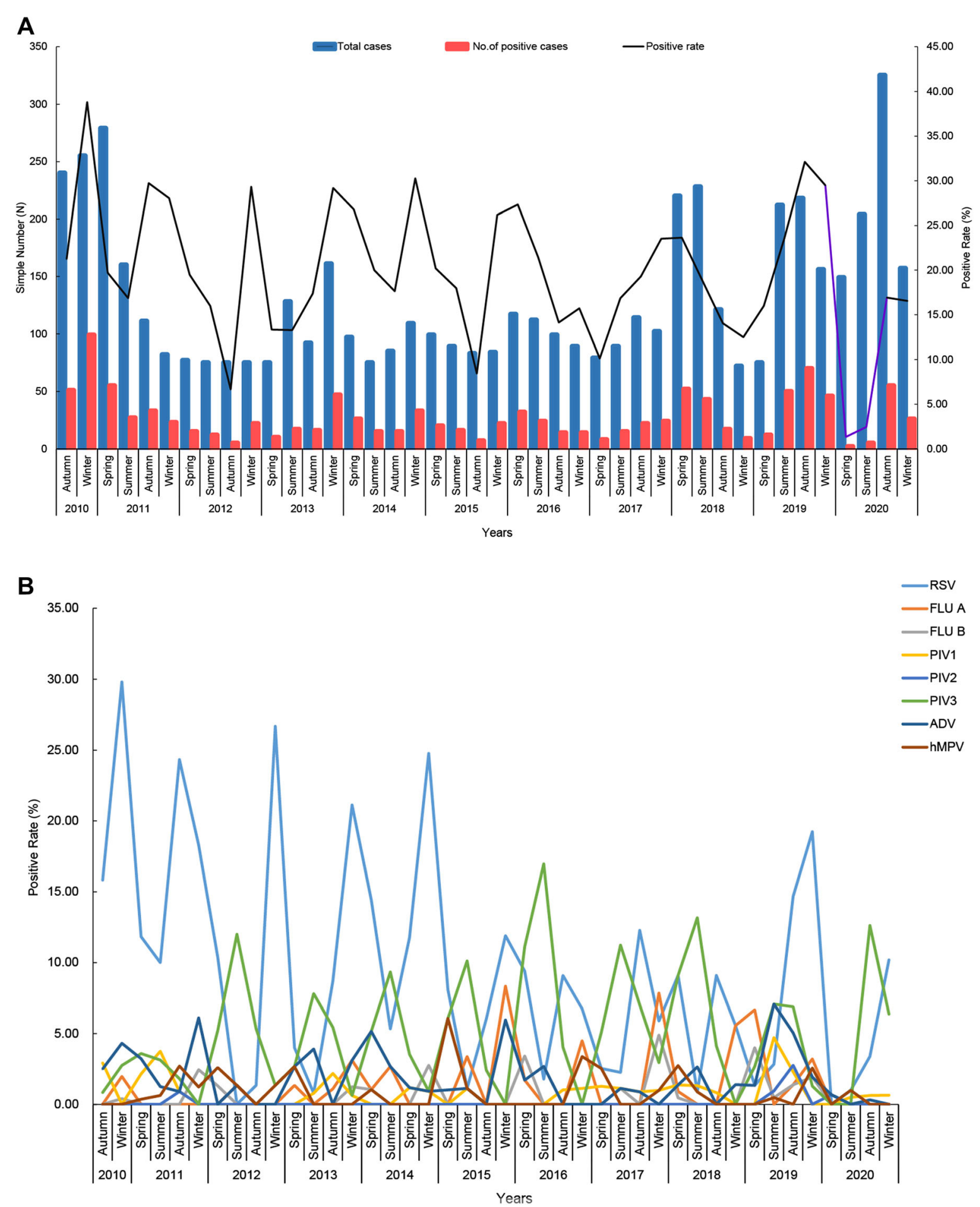

Fig. 2 A and $\mathbf{B}$ Trend of the detection rate for eight respiratory viruses in different seasons. We present the distribution of the sample number and positive samples in each season for the past 10 years. $\mathbf{A}$ and $\mathbf{B}$ Total positive rates of eight viruses in each season and the positive rates

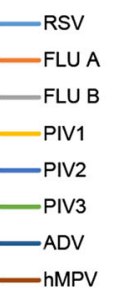

of each virus. The purple broken line on the right of A represents the change in the total detection rate for eight viruses in 2020 
following year), except 2018. In contrast, PIV3 showed a high detection rate in summer (June to August) each year (Fig. 2B).

In terms of yearly distribution, we found the annual total detection rate for the eight viruses showed a slight fluctuation during 2011-2018 (Table 2). This fluctuation of the total detection rate was mainly caused by the change in RSV. From 2011 to 2018, the total detection rate for the eight viruses ranged from $22.6 \%(83 / 366)$ to $16.8 \%(51 / 302)$, with the lowest total detection rate in 2012. After 5 consecutive years of high annual detection rate of RSV (9.6-23.0\%) from 2010 to 2014, 4 consecutive years of low annual detection rate of RSV (5.8-6.8\%) from 2015 to 2018 followed. The total detection rate in 2019 reached $26.9 \%(178 / 661)$, probably because of the combined high prevalence of RSV, PIV3 and ADV during that year. In 2020, the detection rate of each virus except PIV3 dropped dramat- ically, and the overall detection rate dropped to $10.5 \%(88 / 835)$, which was the lowest in the past 10 years (Fig. 2, Table 2). As the 2010 specimens were collected between September and December, the overall detection rate for that year was $30.3 \%(150 / 495)$.

SARS-CoV-2 was found in Wuhan, China, in November 2019. COVID-19 spread across China rapidly, peaking in February 2020 ( $>69,500$ cases). Since August 2020, when Chinese communities reopened, the number of patients with respiratory and enterovirus infections (represented by HFMD and herpangina) has gradually increased. The detection rate of respiratory viruses, mainly RSV and PIV3, grew gradually with decreasing temperature, but the influenza virus still almost disappeared. Due to the period from February to July 2020, the detection rate of the eight respiratory viruses was close to zero; the total detection rate of the eight viruses in

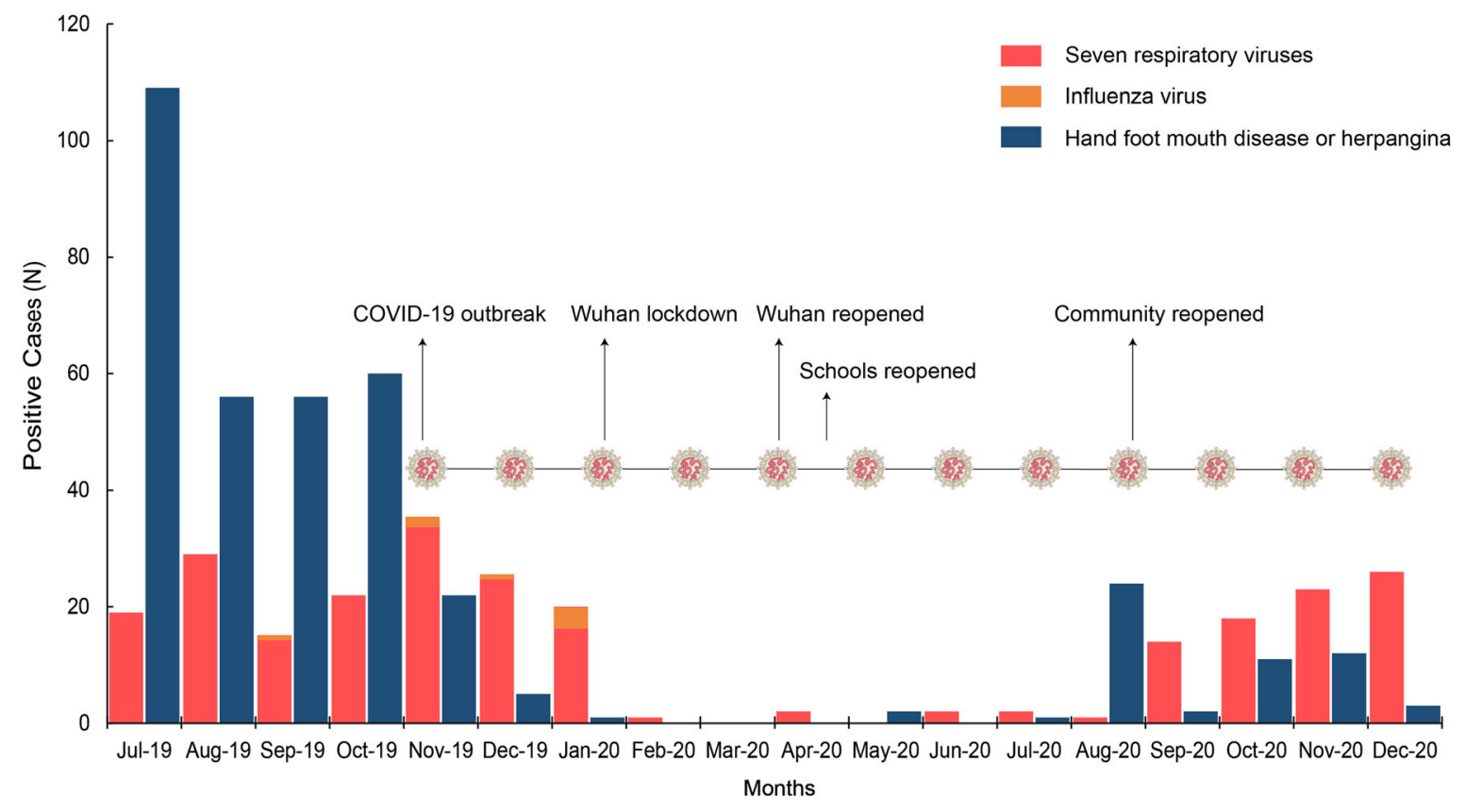

Fig. 3 Between July 2019 and December 2020, the monthly distribution of positive cases of eight respiratory viruses (including influenza viruses) among the 5544 patients enrolled in this study and confirmed cases of HFMD and herpangina in our clinical center were analyzed. Since January 2020, Chinese residents in communities have been required to wear facemasks, keep social distance and pay attention to hand hygiene. Since February 2020, COVID-19 has changed to a sporadic epidemic in China, and most cases were imported 
2020 showed a significant decline for the first time in the past 10 years (Table 2, Figs. 2 and 3). This resulted in a typical "V" shape for the 2020 broken line pattern in the 10-year trend of total virus detection rates for the eight viruses (Fig. 2A).

\section{DISCUSSION}

In this retrospective study, the total detection rate of eight respiratory viruses by DFA was $20.3 \%$, especially the detection rate of $17.9 \%$ in severe CAP cases, which was far lower than the results of our previous studies using molecular biological methods [13-15]. We believe this was related to the low sensitivity of DFA to nucleic acid detection methods. However, this study clearly showed the seasonal characteristics of the epidemics of eight viruses in children with CAP over the past 10 years. For example, RSV detection rates were highest in children with respiratory infections in winter, especially in younger children, as reported $[12,16]$.

From 2011 to 2019 , the total detection rate of the eight viruses was relatively stable, but a dramatic change occurred in 2020. It was a unique opportunity for us to learn about disease control in communities outside of health facilities. This unique opportunity allowed us to rehearse the preventive and therapeutic strategies for the second " $\mathrm{X}$ " disease pandemic in the future. We also experienced the impact of changes in the behavior patterns of the whole population on the prevalence of pathogens circulating among the public.

Sustained community-based measures to prevent COVID-19 transmission included keeping social distance, using facemasks, carrying out hand hygiene campaigns and closing schools or communities, and even cities, in China. Figure 3 shows the change of positive rates for the eight viruses was highly consistent with the timeline of disease control measures being carried out. Not only the positive rates of respiratory viruses, but also those of enterovirus infection cases, such as HFMD or herpangina, were close to zero in our clinical center, which had never happened for these respiratory viruses in the past 10 years. This phenomenon was demonstrated by a marked decline in the incidence of enterovirus encephalitis following the start of strict COVID-19 quarantine measures, but not only in the incidence of respiratory viruses $[17,18]$. We also observed a resurgence of cases of respiratory and enterovirus infections after the reopening of schools and communities. As was observed in Israel and England with the reopening of schools, the number of COVID-19 cases in children has increased again $[19,20]$.

Our study has some limitations: Our clinical center is the unique treatment center for children with SARS-CoV-2 in the region as stipulated by the Shanghai Municipal Government. One consequence of this is a significant decline in visits by infectious pediatric patients because of the public's worries, which may also limit the credibility of this single-center study in truly reflecting the detection of eight viruses related to pediatric CAP in 2020. Also, our clinical center is a tertiary children's hospital, and the cases received may be more serious. In this study, $12.5 \%$ of the patients suffered from severe pneumonia and $30.6 \%$ of the cases had underlying diseases, indicating the existence of that background. This also restricted our study, which might not be able to fully reflect the actual detection rates of eight viruses in CAP children in Shanghai, especially as we did not enroll all hospitalized CAP patients in our clinical center during the 10 years.

\section{CONCLUSIONS}

These eight respiratory viruses, especially RSV and PIV, were important pathogens of CAP in Shanghai children in the past 10 years. Individual and social infection control measures triggered by the COVID-19 pandemic have had a significant impact on the detection rate of these eight respiratory viruses as well as a definite impact on the prevalence of enteroviruses. This unique opportunity allowed us to rehearse the control strategies control and preventive measures for regular and emerging respiratory viruses, even the second " $\mathrm{X}$ " disease pandemic in the future. 


\section{ACKNOWLEDGEMENTS}

We are grateful for the support provided by the Chinese CDC and Wuhan Institute of Virology.

Funding. This work was partially supported by the Shanghai Municipal Commission of Health and Family Planning (grant 201840282) and the Shanghai Science and Technology Commission Medical Project (grant 19411972100). The journal's Rapid Service Fee was funded by the authors.

Authorship. All named authors meet the International Committee of Medical Journal Editors (ICMJE) criteria for authorship for this article, take responsibility for the integrity of the work as a whole, and have given their approval for this version to be published.

Author Contributions. Jun Shen and Wenjie Tan conceptualized and designed the study and reviewed and revised the final manuscript. Fei Li and Yuhan Zhang collected clinical data, analyzed the results, and drafted the initial manuscript. Peng Shi, Linfeng Cao and Liyun Su analyzed the results provided clinical context for study methods; Yulan Zhang, Ke Peng and Roujian Lu critically contributed to the final manuscript. All authors approved the final manuscript as submitted and agree to be accountable for all aspects of the work.

Disclosures. Fei Li, Yuhan Zhang, Peng Shi, Linfeng Cao, Liyun Su, Yulan Zhang, Ke Peng, Roujian Lu, Wenjie Tan and Jun Shen all confirm that they have no conflicts of interest to declare.

Compliance with Ethics Guidelines. This study involving human participants were reviewed and approved by the medical ethics committee of the Children's Hospital of Fudan University (2021-292).

Data Availability. The datasets analyzed during the current study are not publicly available due to the reason that these data came from a long-term surveillance but are available from the corresponding author on reasonable request.

Open Access. This article is licensed under a Creative Commons Attribution-NonCommercial 4.0 International License, which permits any non-commercial use, sharing, adaptation, distribution and reproduction in any medium or format, as long as you give appropriate credit to the original author(s) and the source, provide a link to the Creative Commons licence, and indicate if changes were made. The images or other third party material in this article are included in the article's Creative Commons licence, unless indicated otherwise in a credit line to the material. If material is not included in the article's Creative Commons licence and your intended use is not permitted by statutory regulation or exceeds the permitted use, you will need to obtain permission directly from the copyright holder. To view a copy of this licence, visit http://creativecommons.org/licenses/by$\mathrm{nc} / 4.0 /$.

\section{REFERENCES}

1. Lee $\mathrm{H}$, Lee $\mathrm{H}$, Song $\mathrm{KH}$, et al. Impact of public health interventions on seasonal influenza activity during the SARS-CoV-2 outbreak in Korea. Clin Infect Dis. 2021;73(1):e132-140.

2. Dyrdak R, Hodcroft EB, Wahlund M, Neher RA, Albert J. Interactions between seasonal human coronaviruses and implications for the SARS-CoV-2 pandemic: a retrospective study in Stockholm, Sweden, 2009-2020. J Clin Virol. 2021;136:104754.

3. Poole S, Brendish NJ, Clark TW. SARS-CoV-2 has displaced other seasonal respiratory viruses: results from a prospective cohort study. $\mathrm{J}$ Infect. 2020;81(6):966-72.

4. Soo RJJ, Chiew CJ, Ma S, Pung R, Lee V. Decreased influenza incidence under COVID-19 control measures, Singapore. Emerg Infect Dis. 2020;26(8): 1933-5.

5. Kuo SC, Shih SM, Chien LH, Hsiung CA. Collateral benefit of COVID-19 control measures on influenza activity, Taiwan. Emerg Infect Dis. 2020;26(8): 1928-30. 
6. Redlberger-Fritz M, Kundi M, Aberle SW, Puchhammer-Stöckl E. Significant impact of nationwide SARS-CoV-2 lockdown measures on the circulation of other respiratory virus infections in Austria. J Clin Virol. 2021;137:104795.

7. Wang Y, Tian H, Zhang L, et al. Reduction of secondary transmission of SARS-CoV-2 in households by face mask use, disinfection and social distancing: a cohort study in Beijing, China. BMJ Glob Health. 2020;5(5): e002794.

8. Petersen E, Koopmans M, Go U, et al. Comparing SARS-CoV-2 with SARS-CoV and influenza pandemics. Lancet Infect Dis. 2020;20(9):e238-244.

9. Hsiang S, Allen D, Annan-Phan S, et al. The effect of large-scale anti-contagion policies on the COVID19 pandemic. Nature. 2020;584(7820):262-7.

10. To KK, Sridhar S, Chiu KH, et al. Lessons learned 1 year after SARS-CoV-2 emergence leading to COVID-19 pandemic. Emerg Microbes Infect. 2021;10(1):507-35.

11. Braithwaite I, Callender T, Bullock M, Aldridge RW. Automated and partly automated contact tracing: a systematic review to inform the control of COVID19. Lancet Digit Health. 2020;2(11):e607-621.

12. Bradley JS, Byington CL, Shah SS, et al. The management of community-acquired pneumonia in infants and children older than 3 months of age: clinical practice guidelines by the Pediatric Infectious Diseases Society and the Infectious Diseases Society of America. Clin Infect Dis. 2011;53(7):e2576.

13. Zhao Y, Shen J, Wu B, et al. Genotypic diversity and epidemiology of human rhinovirus among children with severe acute respiratory tract infection in
Shanghai, 2013-2015. Front Microbiol. 2018;9: 1836.

14. Wang Y, Li Y, Liu J, et al. Genetic characterization of human bocavirus among children with severe acute respiratory infection in China. J Infect. 2016;73(2):155-63.

15. Li F, Wang Y, Zhang Y, et al. Etiology of severe pneumonia in children in alveolar lavage fluid using a high-throughput gene targeted amplicon sequencing assay. Front Pediatr. 2021;9:659164.

16. Fitzpatrick T, McNally JD, Stukel TA, et al. Family and child risk factors for early-life RSV illness. Pediatrics. 2021;147(4): e2020029090.

17. Luciani L, Ninove L, Zandotti C, Nougairède A. COVID-19 pandemic and its consequences disrupt epidemiology of enterovirus meningitis South-East France. J Med Virol. 2021;93(4):1929-31.

18. Kies KD, Thomas AS, Binnicker MJ, Bashynski KL, Patel R. Decrease in enteroviral meningitis: an unexpected benefit of COVID-19 mitigation? Clin Infect Dis. 2020. https://doi.org/10.1093/cid/ ciaa 1881.

19. Somekh I, Shohat T, Boker LK, Simões EAF, Somekh E. Reopening schools and the dynamics of SARSCoV-2 infections in Israel: a nationwide study. Clin Infect Dis. 2021. https://doi.org/10.1093/cid/ ciab035.

20. Mensah AA, Sinnathamby M, Zaidi A, et al. SARSCoV-2 infections in children following the full reopening of schools and the impact of national lockdown: prospective, national observational cohort surveillance, July-December 2020, England. J Infect. 2021;82(4):67-74. 